\title{
On the applicability of the Ivantsov growth equation
}

\author{
Zi-Kui Liu ${ }^{\mathrm{a})}$ and $\mathrm{Y}$. Austin Chang \\ Department of Materials Science and Engineering, 1509 University Avenue, \\ University of Wisconsin-Madison, Madison, Wisconsin 53706
}

(Received 24 May 1997; accepted for publication 22 July 1997)

\begin{abstract}
The steady state growth equation for a paraboloid of revolution and a parabolic cylinder, taking the interfacial energy into account, is revisited. Although the consideration of the interfacial energy was necessary, the growth equation was much more complicated than the original equation with a zero interfacial energy, since both the thermodynamics and kinetics of the growth are considered in one equation. In the present work, we take advantage of the development in computational thermodynamics and consider the thermodynamics and kinetics of the process in separate equations, but solve them simultaneously. A consistent framework to describe the phenomena and compare it with previous treatment of the interfacial energy will be presented. (C) 1997 American Institute of Physics. [S0021-8979(97)04321-1]
\end{abstract}

\section{INTRODUCTION}

The exact steady state growth equations for a paraboloid of revolution and a parabolic cylinder were derived by Ivantsov, ${ }^{1}$ and by Horvay and Cahn, ${ }^{2}$ under the assumption that the composition of the matrix in contact with the growing phase is constant along the interface. It was pointed out that due to the interfacial curvature and interfacial kinetics, the interface is essentially nonisoconcentrational for an isothermal transformation ${ }^{3,4}$ and nonisothermal in solidification. 5 Approximate treatments were presented therein.

Further detailed modifications were carried out by Trivedi and co-workers ${ }^{7,8}$ to take the interfacial curvature and finite interfacial mobility (interfacial kinetics) into account. The modification was done by first considering the effects of the interfacial kinetics and the interfacial curvature on the composition close to the interface, and then solving the diffusion equations. Although the modification was necessary, the Trivedi et al.'s equation was more complicated than the original Ivantsov equation, mainly due to the fact that the thermodynamics and kinetics of the process are considered in one equation, which inevitably introduces a number of approximations.

In a recent publication by $\mathrm{Liu},{ }^{9}$ the Ivantsov equation was then used directly with the actual composition close to the interface after taking into account interfacial curvature, finite interfacial mobility, solute drag, and coherency strain energy. The basic idea was to treat thermodynamics and kinetics separately, then couple them together by solving all equations simultaneously by a numeric method. In this manner, all kinetic equations retained their simple forms even when complex situations were considered. A similar usage of the Ivantsov equation was also conducted by Warren and Boettinger. ${ }^{10}$ On the other hand, if the procedure used by Trivedi was followed, the growth equation would become very complicated, and would have had to be modified every time a new effect on the growth kinetics was introduced. However, it is not apparent if the direct usage of the Ivantsov

${ }^{a)}$ Electronic mail: zikui@msae.cae.wisc.edu equation will give reasonable results in comparison with Trivedi's modification. It thus seems desirable to explore this problem further.

In the present article, some detailed comparisons are given. The isothermal transformation from $\gamma(\mathrm{fcc})$ to $\alpha$ (bcc) in the binary $\mathrm{Fe}-\mathrm{C}$ system as used in a previous publication ${ }^{9}$ will be considered. For simplicity, only the interfacial curvature will be considered for a steady state shape with the geometry shown schematically in Fig. 1 and the concentration profile in Fig. 2. For transformations concerning sidebranching and marginal stability theory, e.g., free dendritic growth, the readers are referred to the review by Glicksman ${ }^{11}$ and references therein. For transformation without sidebranching and the maximum growth rate hypothesis, the readers are referred to the work by $\mathrm{Liu}^{12}$ and the references therein.

\section{IVANTSOV EQUATION AND TRIVEDI'S MODIFICATION}

Only the Ivantsov equation and Trivedi's modification will be discussed in the present work, and applied to the $\gamma \Rightarrow \alpha$ transformation in the $\mathrm{Fe}-\mathrm{C}$ system. The derivations of the two equations will not be presented, but referred to the original publications.

The Ivantsov equation has the form

$$
\Omega_{0}=\sqrt{\pi \operatorname{Pe}^{P}}(1-\operatorname{erf} \sqrt{P}),
$$

where $P=v \rho / 2 D_{C}^{\gamma}$, the so-called Peclet number, with $v$ being the interface velocity, $\rho$ the tip radius, and $D_{C}^{\gamma}$ the diffusivity of carbon in the $\gamma$ phase. $\Omega_{0}$ is the nondimensional supersaturation defined as

$$
\Omega_{0}=\frac{u^{0}-u^{\gamma}}{u^{\alpha}-u^{\gamma}},
$$

$u^{0}, u^{\alpha}$, and $u^{\gamma}$ are various carbon mole fractions (see Fig. 2) expressed per mole of substitutional elements: the so-called $u$ fraction. In this case, $\mathrm{Fe}$ is the only substitutional in the present case. This type of concentration is useful when the 


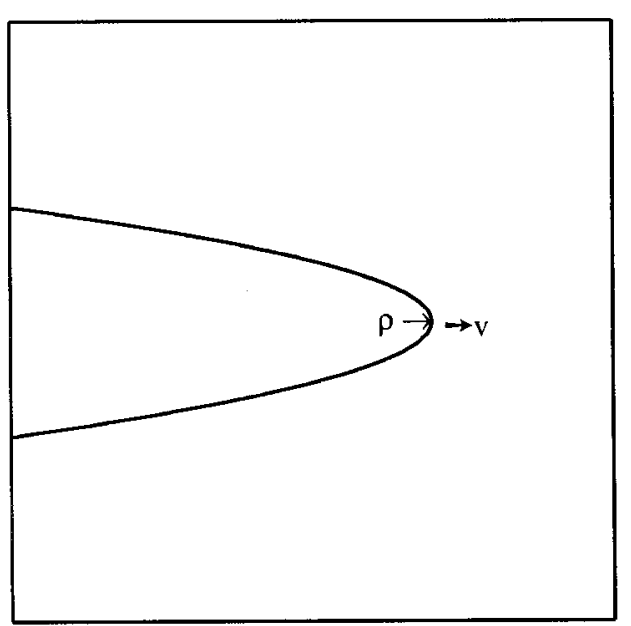

Growth Direction

FIG. 1. Schematic geometry of the system.

interstitial element's contribution to the volume is small. erf is the error function, and $\operatorname{erf} \sqrt{P}=1-\operatorname{erfc} \sqrt{P}$. The right-hand side form was used by Trivedi.

In the Ivantsov equation, $u^{\alpha}$ and $u^{\gamma}$ are obtained directly from phase diagrams for a given temperature, i.e., full local equilibrium is assumed at the interface. However, it is known that the phase compositions at a curved interface with nonzero interfacial energy will differ from those read from a phase diagram, according to the Gibbs-Thompson equation.

Trivedi and Pound ${ }^{7}$ introduced the effect of interfacial curvature on the equilibrium composition of the parent phase by assuming that the equilibrium composition of the growing phase was not affected, and solving the diffusion equation in a manner similar to Ivantsov, but with the new composition. The following equation was obtained under the assumptions of a steady-state parabolic interface near the growing tip and a dilute solution of the parent phase:

$$
\Omega_{0}=\sqrt{\pi \operatorname{Pe}^{P}}(1-\operatorname{erf} \sqrt{P})\left(1+\frac{\rho_{c}}{\rho} \Omega_{0} S_{2}(P)\right),
$$

where $\rho_{c}$ is the critical radius for growth, $S_{2}(P)$ is a complex function of $P,{ }^{8}$ and was approximated by Trivedi ${ }^{13}$ as $2 / \pi P$. The difference between the Ivantsov equation and Trivedi's modification is the extra term in the large bracket in the above equation.

Since except for the boundary condition both Ivantsov and Trivedi solved the diffusion equation in similar ways, it is thus tempting to use the original Ivantsov equation directly, but with the compositions at the interface given by the Gibbs-Thompson equation, if one is only interested in the growth at the tip, i.e.,

$$
\Omega_{\rho}=\sqrt{\pi \operatorname{Pe}^{P}}(1-\operatorname{erf} \sqrt{P}),
$$

where $\Omega_{\rho}$ has the same form as $\Omega_{0}$ in Eq. (2), but $u^{\alpha}$ and $u^{\gamma}$ are the compositions after the interfacial curvature is taken into account. Indeed, Trivedi and Pound ${ }^{7}$ presented an equation similar to the above with the right hand side multiplied

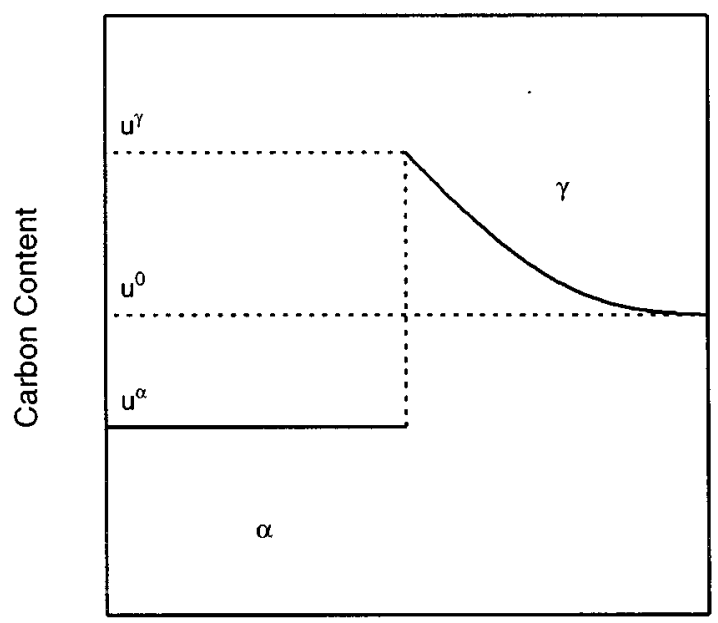

Distance

FIG. 2. Schematic concentration profile across the interface.

by a complex correction factor [see their Eq. (24)]. To date, there appear to have been no detailed examinations of the importance of the correction factor. This will be now discussed by comparing the calculations using Eqs. (3) and (4), respectively.

\section{CALCULATION RESULTS AND DISCUSSION}

There are four unknowns involved in the calculation: the two carbon contents, $u^{\alpha}$ and $u^{\gamma}$; the interface velocity, $v$; and the tip radius, $\rho$. Values of $u^{\alpha}$ and $u^{\gamma}$ are calculated from the thermodynamic properties of the $\alpha$ and $\gamma$ phases for a given tip radius. Since there is only one equation relating $v$ and $\rho$, no unique values for $v$ and $\rho$ exist. As discussed recently $^{12}$ the maximum growth rate principle suggested by Zener, ${ }^{3}$ that a precipitate would spontaneously adjust its shape until it has reached its maximum growth rate, may be applicable to growth without sidebranching. Reasonable agreement has been previously shown between experimental results and calculations. ${ }^{9}$

Prior to carrying out detailed calculations, a brief description of the computational thermodynamics technique is presented. In this technique, the free energies of individual phases in a system are evaluated from theoretical and experimental information on the thermochemistry and phase equilibrium, to form a consistent thermodynamic description of the system. As the free energy is one of the fundamental thermodynamic functions, all kinds of thermodynamic quantities, not limited to the equilibrium quantities, can be readily calculated when the thermodynamic description is available. With the development of modern computer programs, this technique can now handle more and more complicated systems.

The thermodynamic description of the $\mathrm{Fe}-\mathrm{C}$ system was obtained by this technique by Gustafson. ${ }^{14}$ Using this description, the assumption of a dilute solution is no longer 


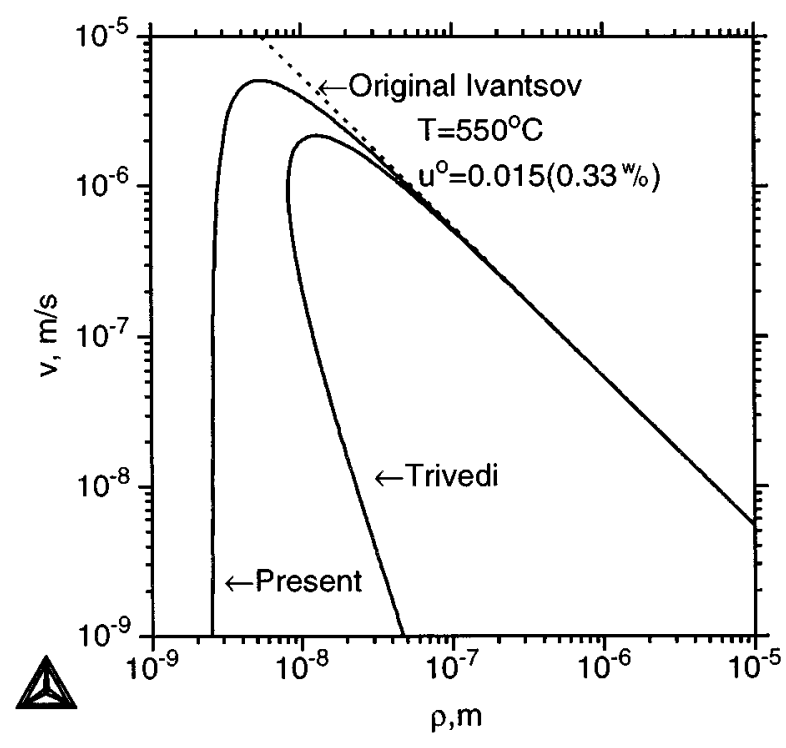

FIG. 3. Calculations for $u^{0}=0.015$ with growth rate on the vertical axis and plate edge radius on the horizontal axis, both in logarithmic scale, by the modified Ivantsov equation and the Trivedi's modification, respectively.

necessary. For a curved interface, equilibrium conditions at the interface may be written as:

$$
\begin{aligned}
& \mu_{\mathrm{C}}^{\gamma}-\mu_{\mathrm{C}}^{\alpha}=\frac{2 \sigma}{\rho} V_{\mathrm{C}}, \\
& \mu_{\mathrm{Fe}}^{\gamma}-\mu_{\mathrm{Fe}}^{\alpha}=\frac{2 \sigma}{\rho} V_{\mathrm{Fe}},
\end{aligned}
$$

where $\mu_{\mathrm{C}}^{\gamma}, \mu_{\mathrm{C}}^{\alpha}, \mu_{\mathrm{Fe}}^{\gamma}$, and $\mu_{\mathrm{Fe}}^{\alpha}$ are the chemical potentials of $\mathrm{C}$ and $\mathrm{Fe}$ in $\alpha$ and $\gamma$ at the interface, respectively. $\sigma$ is the interfacial energy and its value is chosen to be $0.2 \mathrm{~J} / \mathrm{m}^{2} . V_{\mathrm{C}}$ and $V_{\mathrm{Fe}}$ are the partial molar volumes of $\mathrm{C}$ and $\mathrm{Fe}$, respectively, assuming the same values for both $\alpha$ and $\gamma$. As the carbon atoms occupy interstitial sites, they are assumed to have no contribution to the volume; $V_{\mathrm{C}}=0$ and $V_{\mathrm{Fe}}=7$ $\times 10^{-6} \mathrm{~m}^{3} / \mathrm{mol} . u^{0}=0.015$, i.e., $0.33 \mathrm{wt} \%$ and $T=550{ }^{\circ} \mathrm{C}$ are used, as in previous work, ${ }^{9}$ which gives $\rho_{c}=1.992$ $\times 10^{-9} \mathrm{~m}$. The calculations were carried out using the PARROT program $^{15}$ in THERMO-CALC. ${ }^{16}$

Figure 3 depicts the relation between the velocity $(v)$ and tip radius $(\rho)$ for the original Ivantsov equation [Eq. (1)], the modified Ivantsov equation [Eq. (4)], and Trivedi's equation [Eq. (3)]. As expected at large tip radii, they give identical values because the contribution from the interfacial curvature is small. They begin to differ from each other at about $\rho=10^{-7}(\mathrm{~m})$. The original Ivantsov equation, which did not take interfacial curvature into account, gives high velocity (dotted line) and will not be discussed further.

The maximum velocities are $5.0943 \times 10^{-6}$ and 2.2247 $\times 10^{-6}(\mathrm{~m} / \mathrm{s})$ for the modified Ivantsov and Trivedi's equations at the tip radii of $5.4 \times 10^{-9}$ and $1.2 \times 10^{-8}(\mathrm{~m})$, respectively. It is noted that the left part of the curve for Trivedi's equation bends back to larger tip radii with decreasing velocity. This may be due to the dilute solution assumption used by Trivedi and Pound. Figure 4 shows that the calcu-

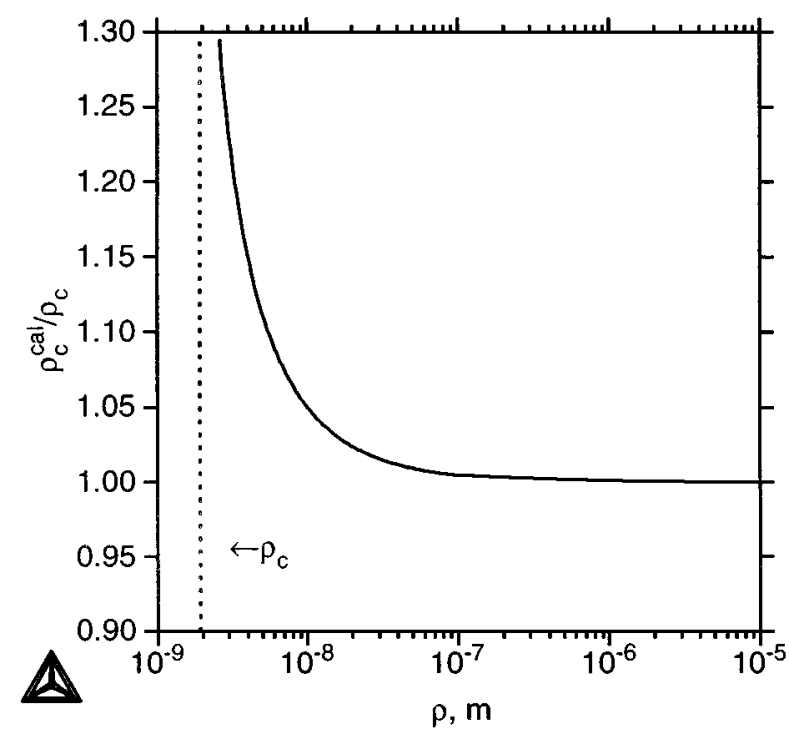

FIG. 4. The ratio of the calculated critical radius with the dilute solution assumption over the critical radius plotted with respect to the tip radius.

lated critical radius from the dilute solution is not constant when the tip radius is below about $10^{-7}(\mathrm{~m})$, with

$$
\rho_{c}^{\mathrm{cal}}=\frac{u^{r 0}-u^{r}}{u^{r 0}-u^{0}} \rho
$$

derived from the equations by Trivedi, ${ }^{8}$ where $u^{r 0}$ is the composition with a planar interface.

It is interesting to note that in studying the solidification of a pure component, Nash and Glicksman ${ }^{17}$ found that the maximum growth velocity from their calculation is about 2.28 times the velocity from Trivedi's equation (see Ref. 18). In the present example, the ratio of the maximum velocity obtained to that calculated from Trivedi's equation is $5.0943 / 2.2247=2.29$. These results suggest that we may thus use the Ivantsov equation directly with the actual compositions at the interface obtained from computational thermodynamics.

The advantage in using the Ivantsov equation directly is self-evident. From Eq. (3), it is clear that, if one would like to consider effects other than interfacial curvature, one would have to solve the diffusion equation over again, and obtain another complex correction term on the right-hand side of Eq. (3), as shown by Trivedi ${ }^{8}$ for inclusion of interfacial kinetics. With the present approach, it is not necessary to modify the Ivantsov equation. Instead, we can simply use the equilibrium compositions at the phase interface obtained from computational thermodynamics as has been done earlier for finite interfacial mobility, elastic stresses, and solute drag. ${ }^{9}$

\section{SUMMARY}

The modified Ivantsov equation is compared with Trivedi's modification by considering the interfacial curvature with nonzero interfacial energy. Due to modern computational thermodynamic techniques, the dilute solution assumption is not needed. It is demonstrated that it is reason- 
able to use the Ivantsov equation directly with the modified interfacial compositions. Consequently, no extra complications will be anticipated when more complicated situations are considered, as shown in previous work ${ }^{9}$ in which the interfacial curvature, finite interfacial mobility, solute drag, and coherency energy were considered in a unified and selfconsistent framework.

It may further be postulated that kinetic equations derived for individual single processes can be combined to describe a complex process through coupling with computational thermodynamics.

\section{ACKNOWLEDGMENTS}

The Thermo_Calc program licensed from The Foundation for Computational Thermodynamics is used for all calculations and is greatly appreciated. The authors would like to thank John Agren for his comments on the manuscript and John Woodford for carefully reading the manuscript. They also wish to acknowledge the partial financial support of the National Science Foundation through Grant No. NSF-DMR94-21780, and Dr. Bruce MacDonald of the Metal Program of the Materials and Processing Cluster of NSF for his interest in this work.

${ }^{1}$ G. P. Ivantsov, Dokl. Akad. Nauk SSSR 58, 567 (1947).

${ }^{2}$ G. Horvay and J. W. Cahn, Acta Metall. 9, 695 (1961).

${ }^{3}$ C. Zener, Trans. AIME 167, 550 (1946).

${ }^{4}$ M. Hillert, Jernkontorets Ann. 141, 757 (1957).

${ }^{5}$ D. E. Temkin, Dokl. Akad. Nauk SSSR 132, 1307 (1960).

${ }^{6}$ G. F. Bolling and W. A. Tiller, J. Appl. Phys. 32, 2587 (1961).

${ }^{7}$ R. Trivedi and G. M. Pound, J. Appl. Phys. 40, 4293 (1969).

${ }^{8}$ R. Trivedi, Metall. Trans. 1, 921 (1970).

${ }^{9}$ Z.-K. Liu, Acta Mater. 44, 3855 (1996).

${ }^{10}$ J. A. Warren and W. J. Boettinger, Acta Metall. Mater. 43, 689 (1995).

${ }^{11}$ M. E. Glicksman, Mater. Sci. Eng. 65, 45 (1984).

${ }^{12}$ Z.-K. Liu, J. Appl. Phys. 71, 4809 (1992).

${ }^{13}$ R. Trivedi, Solid-Solid Phase Transformation, Proceedings of the International Conference (The Metallurgical Society of AIME, New York, 1982), pp. 477-502.

${ }^{14}$ P. Gustaffson, Scand. J. Metall. 14, 632 (1985).

${ }^{15}$ B. Jansson, Trita-Mac-0234 (Royal Institute of Technology, Stockholm, Sweden, 1984).

${ }^{16}$ B. Sundman, B. Jansson, and J.-O. Andersson, CALPHAD: Comput. Coupling Phase Diagrams Thermochem. 9, 153 (1985).

${ }^{17}$ G. E. Nash and M. E. Glicksman, Acta Metall. 22, 1283 (1974).

${ }^{18}$ M. E. Glicksman, R. J. Schaefer, and J. D. Ayers, Metall. Trans. A 7, 1747 (1976). 